\title{
The contribution of the Capability Approach to demographic analysis: lessons learned. Tracking Inequalities.
}

\author{
Claudine Sauvain-Dugerdil' and Allan G.Hill \\ ${ }^{1}$ Institute of Demographic and Life course studies (I-DEMO), University of Geneva, Geneva, \\ Claudine.Sauvain@unige.ch. \\ ${ }^{2}$ Harvard School of Public Health (Boston) and University of Southampton (UK).
}

\begin{abstract}
In this concluding paper, we discuss the contribution of the Capability Approach to the "tracking" of inequalities, i.e. focusing on opportunities rather than outcomes and targeting both resources and the means to use these resources. We return to two central dimensions of our analyses: the multiple nature of well-being and the different kinds of means that modulate the unequal individual ability to live a life of quality. We summarize our main results regarding the nature of health and its determinants, the function of services and the multiple meanings of occupation, as well as the role of contextual resources, individual endowments and acquired capacities. A third dimension concerns the role of the global context and what can be said in particular about differences between Mali and Ghana. In the last part, we discuss further developments to improve the tracking of inequalities, first through cross-cutting analyses of different sources of vulnerability and secondly, by making allowance for individual agency.
\end{abstract}

Keywords: Capability approach, inequalities, agency, Mali, Ghana.

\section{Résumé}

\section{La contribution de l'approche des Capabilités à l'analyse démographique: leçons à tirer. Traquer les inegalites}

Nous discutons dans cet article de conclusion de la contribution de l'approche des Capabilités pour traquer les inégalités, à savoir se centrer sur les opportunités plutôt que le résultat et cibler les ressources et les moyens de les utiliser. Nous revenons sur deux dimensions centrales de nos analyses : la nature multiple du bien-être et les inégalités de capacité à vivre une vie de qualité. Nous résumons nos principaux résultats sur la nature de la santé et de ses déterminants, la fonction des services et les significations multiples de l'activité professionnelle, ainsi que le rôle des ressources du contexte et des caractéristiques individuelles. Une troisième dimension concerne le contexte global et les différences entre le Mali et le Ghana. Dans la dernière partie, nous suggérons des développements pour mieux traquer les inégalités, d'une part, en croisant diverses sources de vulnérabilité et, d'autre part, en intégrant le rôle de la personne.

Mots-clés: Capabilités, inégalités, agency, Mali, Ghana

Applying the Capability Approach (CA), as the preceding papers highlight, is not an easy task, especially with data collected without this analytic approach in mind. One aim of this Supplement is to demonstrate that there are several ways, not a single canonical approach, to make use of the Capability framework, depending on the issue under investigation and the available information. The main lesson learned by these attempts to apply the 
Capability Approach in demographic analysis is that it offers an original and theoretically-based perspective from which to consider population issues and a framework to structure the analysis. As the papers show, there is no single template which will suit all cases but the overall schema seems to have merit and utility. The examples here set out some of the difficulties in measuring behaviour and identifying proxies for the sources of vulnerability at different levels. The CA, we assert, makes a contribution to the progress of a new demography aimed more strategically at the understanding and interpretation of demographic behaviour and sources of inequalities rather than the more common descriptive and comparative approaches. Covering the full range of population issues - family and household composition, morbidity, longevity, mortality and mobility - these topics when viewed through the Capability lens, are regarded as dimensions of people's well-being rather than as end points, and can thus be interpreted as measures of the processes that pave the way to understanding the more complex concept at the centre of the CA - the "freedom to achieve".

Focusing on the person's Capabilities - i.e. "her freedom to live the life she has reason to value" - forces us to consider the array of real opportunities to be and to do and their unequal outcomes measured as achievements achievements resulting from the resources available and the ability and readiness to use them. We agree with Nussbaum that there are basic values, as expressed in her list of capabilities that fits with principles of justice and the rights-based movement, and with Sen that there is not a universal closed list of capabilities nor a unique method of application. As Nussbaum (2006) stresses, the CA is a way to "extend justice to all world citizens" by providing "basic entitlement for all" and at the same time respecting "the many ways citizens choose to live" without any compromise on the basic capabilities. Therefore, the CA implicitly takes into account the diversity of individual situations without ignoring norms and values. The focus is therefore on the individual ability to cope with life's hazards, but with individual agency embedded in a multi-layered context.
More than just "targeting" inequalities, the CA aims to "track" them, in other words, to identify their roots. Two conclusions from this project include the need to take into account the multiple dimensions of well-being and the different kinds of means that modulate the unequal individual ability to live a life of quality. We shall return to the main results of our analyses in these two central dimensions below. A third conclusion concerns the role of the global context and what can be said in particular about differences between Mali and Ghana. In the last part of this concluding paper, we discuss further developments to improve the tracking of inequalities, first through cross-cutting studies of different sources of vulnerability and secondly through interpretations and way to make allowance of individual agency.

\section{Capabilities: considering the multi- dimensional nature of the quality of life}

The causes of vulnerability cannot be discerned through the factors associated with a unique outcome usually measured as a single synthetic or composite score. Focusing on opportunities rather than outcomes forces us to capture the multi-dimensionality of well-being and its determinants through a series of indicators reflecting the person's life condition and her susceptibility to different types of risk factors.

We consider the Capability Framework as a structure for choosing the best proxies to measure inequalities in opportunities and their eventual realization, i.e. the dyad Capabilities Functionings. Whenever possible, we have built probabilistic models (regression analysis) to analyse in which respect the persons enjoying specific conditions differ from others and to test the degree to which varying Capabilities influence the probability of the achievement. This approach requires us to perform the analysis in successive steps by first considering the factors of variability of the proxies for Capabilities that, in a second step, become themselves explanatory factors of the achievement. This has been chosen as an alternative to approaches such as better known path analysis which allows less flexibility in the analysis of heterogeneity. 
These points are illustrated with reference to the capture of multiple nature of health inequalities; the role played by the access to services in the Capability scheme; and the function of the individual's type of occupation.

\section{The multi dimension nature of health and of its determinants}

A major contribution of the CA to the study of health differentials is that the outcome accounts for the "ability to have a good health" seen as a valued state (Ariana and Naveed, 2009). Thus, multiple indicators are needed to disentangle health inequalities due to the context, to personal resources, to behaviour or to position towards values, and their varying influence on different dimensions of health.

- The Accra study shows that the determinants and patterns of mental health differ in many regards from those of physical health, in particular the fact that the context endowment i.e. the relative wealth of the city sectors - makes a difference for physical health, whereas household wealth makes a difference to mental health.

- The Bamako youth study highlights that in the context of a high prevalence of malaria, it is worth separating in the analysis the determinants of acute fever from other health injuries. The former provides information on environmental health and preventive behaviour, whereas the latter is related more to personal resources. More subjectively, self-rated health measures introduce both preferences and cultural norms. The results confirm that youth is a relatively healthy period but associated with risky behaviour which is why it is important to approach the analysis of heterogeneity with several health indicators. This enable us to highlight the puzzling roles of resources, i.e. young men from the richer social strata adopting more risky health behaviour and young women from the richest households having more, not fewer, health problems than those from the poorest sector who are also using more the modern health system.

Body weight is clearly a case of complex interactions between resources, life styles and values. In the African countries concerned, valued life styles may play a role in the prevalence of underweight, but it is mainly an indicator of poverty, whereas in overweight and obesity, the role of resources and life styles are mitigated by a collective valuation of large body size. In this case, specific individual resources appear to make some individuals more ambivalent towards accepted norms and therefore more prone to adopt different habits.

\section{Services (health, contraception) as means not capabilities}

As recalled in Chiappero et al (see first paper of this volume), the use of health services may constitute a Capability when people are "effectively able to access quality healthcare" when pursuing the aim of being healthier. This implies therefore that people have the conviction that it will improve their health state.

In our case studies, the use of health services does not appear to represent a Capability. First, it seems to be limited to those in worse health conditions and poorer. The Accra survey shows that the recourse to medical services characterized the most needy, i.e. those in poorer health, both physical and mental, this even more among poorest households. The same is the case among the young people of Bamako. The use of services is associated with boys' poorer self-evaluation. Among girls, those who had had a previous contact with the modern health system have a higher probability of acquiring a subsequent health problem. A more detailed analysis shows also that the previous contact with the health services improves subsequent health only among those women with higher human and economic resources; it seems thus to increase health inequalities. Moreover, using the modern health 
system appears to be more a matter of opportunities of the moment, including contact outside the household, than a real long-term concern for health.

In the same way, the access to family planning appears to have only a relatively weak impact on the ability to manage the family project, as reflected in birth spacing. On the one hand, it has no effect on the duration of the interval between marriage and first birth. On the other hand, its association with longer birth intervals reduces when controlling for individual characteristics. It is not the fact of adopting contraception that counts but the profile of the women who adopt this behaviour. Thus, contraception does not seem to represent, in itself a Capability, but is rather, a means to realize intentions influenced by other factors.

\section{The multiple meanings of occupation}

In the different studies of the present project, occupation has been considered either as an accomplishment - i.e. type of occupation, job security as an indicator of quality of life - or as a Capability, a proxy for the ability to have control over life, but also as a conversion factor, a means to achieve other dimensions of wellbeing.

The results show that occupation and the type of activities have complex effects on health and health seeking behaviour depending on age and the sector of activity. Physical activity, especially in the agriculture sector is associated with a lower risk of obesity. The health of Accra women is positively associated with having an occupation, formal or informal. Among young Bamako people, working outside the household or being in study is associated with more access to services, but professional activity is associated with a poorer health, especially for men, that may also implies environmental risks and risky life styles. The analysis of contraception use shows that the increased contacts with the outside world brought by the professional activity is not verified among those working in agriculture. Even when controlling for personal resources such as education and household wealth, women and men working in the agriculture sector practice less contraception. The association between job security and fewer children or less desire to have another child (substitution hypothesis) appears to vary between sector of activity, household wealth and number of children already born.

These results show that occupation interacts with other personal characteristics which means it may have to be considered as "combined Capability", that is to say "trained or developed traits and abilities, developed, in most cases, in interaction with the social, economic, familial and political environment" (Nussbaum 20II).

\section{Resources and means to use resources}

The unequal ability to "live the life one has reason to value" results not only from the availability and accessibility of contextual resources including services, but also the willingness to use them for one's own sake. The CA frames the analysis by making a fundamental distinction between endowments and so-called conversion factors. It further distinguishes between personal, family and societal resources, as well as between those resources that are fixed and/or stable and those that evolve along with life experience.

In order to understand the roots of inequalities and possible solutions, more refined measurements are necessary. The Capability framework constitutes a tool to structure the analysis of heterogeneity by distinguishing different sources of inequalities. The aim is to highlight factors of vulnerability shared by specific groups of people, what we have proposed to call "endowment groups". Individual agency is thus considered through the varying importance taken by different types of inequalities and their differential impact on opportunities and achievements. Only specific questions on preferences can however really circumscribe the person's space of freedom, both real and perceived.

\section{Individual endowments and acquired personal resources}

As regard individual characteristics - among which we also include those of the household and of socio-cultural belonging - the Capability framework distinguishes the fixed endowment 
from acquired personal resources that are malleable. Thus a distinction is made between personal resources that can be enhanced and those that cannot be modified. The consequences of the latter on well-being are modulated by the characteristics of the context and by their social meanings. The stages in the life course and gender are among central individual endowments that take on meaning and importance through their socio-cultural meanings.

\section{Life transitions}

Ageing is associated with increased frailty as shown by the steady deterioration of the physical health of the Accra women. However, the fact that such age influences disappear among poorer families living in a richer neighbourhood highlights the buffering effect that the context can play. Ageing is also associated with increased competence which is expressed in our analyses in the stability of mental health over the life course, or a better job security among older persons and in turn a greater job vulnerability of young people. The Bamako youth survey shows that younger men adopt less healthy life styles and that the youngest age group of both sexes are more vulnerable to fevers but consider themselves in better health than the older groups. In Mali and Ghana, those under 20 years have a poorer knowledge of contraception and a lower level of use; women who become mothers earlier have shorter birth intervals. As for occupation, it is marital status, more than age, that plays a role: single persons value more highly higher education, whereas marriage appears to be a handicap for job opportunities for those with no schooling.

Motherhood appears to be a life transition with complex consequences. Among the Bamako young women, those already mothers have a higher risk of health problems but in Mali, mothers' body weight is more often in the normal range (neither under- nor over-weight). In Ghana, the poorest women enjoy better mental health when childless. A higher number of children is correlated with poorer physical health but health risks appear less among those with very large families.

\section{Gender inequalities}

The results confirm that gender inequalities are evidenced in multiple ways, which often requires more advanced analyses. Major gender differences are to be found in the health of Bamako youth: the sanitary conditions in the environment affect the young women more, whereas ethnic origin is a cause of heterogeneity among young men. Out of necessity, the poorest women use the modern health services more whereas young men from the best off neighbourhoods adopt behaviour detrimental to their health. Sarkodie et al's analysis (paper 7) show that, in Ghana, gender inequalities in access to the labour market is not erased by education : whatever their level of schooling, women are mainly found in subsistence agriculture or as independent workers (mainly informal) whereas men are more often in commercial agriculture and paid jobs.

Further, a woman's situation appears to be strongly influenced by that of her partner. In both countries, the partner's job stability enhances that of the wife (in Ghana, only in large families) and is positively associated with the number of children. In Accra, having a spouse is associated with better physical health, but only for women of wealthy households living in a poorer sector. Single women still have a handicap regarding access to contraception (effect weaker in Ghana) whereas for men being married only increases knowledge. The unequal share of the burden of every day family care mentionned in women's interviews matches with the fact that the correlation between an increased use of contraception and a higher number of children is only found among women; among men, contraceptive use is not associated with the current number of children, but with ideal family size.

\section{Economic and human resources}

Household wealth, available in all data sets used here, is interpreted as a resource (an endowment in the capability framework), whereas in socio-demographic analysis, it is more readily seen as a conversion factor, that is to say a means to access and use the context 
resources'. The household wealth is associated with more secure female jobs in Ghana (relationship not significant in Mali) and in both countries with a lower number of children. This is confirmed by the study of contraception which shows higher use and, especially in Mali, longer birth intervals. The household wealth does not improve the health of young Bamako people, whereas it is a factor of better mental health of Accra women (their physical health being rather influenced by the wealth of the context). In both countries, better off households experience less underweight but a higher risk of obesity.

In all analyses, education has an influence, even after controlling for household wealth and context resources, but the effect is often complex and combined with other personal resources. In Ghana, higher education does not appear to be enough to secure a better job. In both countries, literate women have fewer children, but they do not differ in terms of the wish of an extra child. The effect of being literate on professional activity varies with the household wealth, the sector of activity and whether the woman already has children. The Accra women survey shows that the absence of schooling, or school drop out after a few years, constitute handicaps for physical and mental health even among the richer households, but relative wealth (being richer among poorer sectors or being poorer in richer in poorer sector) appears to compensate for the deficit in schooling. The $\cap$ shape effect of schooling on the health of young Bamako women and men confirms that access to school may be a risk factor when not compensated by the skill acquired through a longer schooling.

\section{Context resources: three approaches}

Our analyses highlight the important role played by the context of residence even when controlling for individual characteristics. Nonetheless, interpretations are often complex and require a good knowledge of the field.

The different studies have used three different ways to measure the specificities of the surroundings and to use them in the analyses:
- Multi-level analysis. Jacobi (paper 8) has built ten variables ${ }^{2}$ describing the characteristics of the spatial unit used (30 in Ghana and 25 in Mali by crosscutting administrative sub-divisions and urban/rural settings). Results show that the specificities of the context do not modify the effects of individual characteristics, but have their own effects even when controlling for the latter. For instance, in Ghana the wealth of the context appears to increase the wish for another child, whereas in Mali, it negatively correlated with the number of children, such as the percent of women who are household heads. In Ghana, those women living in a context where the average female status is better, have more job security. A higher ethno-linguistic heterogeneity increases the job security of Mali women, and reduces their number of children and, in Ghana, the wish for another child. In short, although we can suggest interpretations for each of these effects, they are not globally consistent and often differ between both countries.

\section{- Specificities of the place of residence} introduced as an individual trait. The analysis of the health of Accra women introduces the wealth of the sector of residence as a characteristic to be crosscut with the family wealth. Some DHS surveys provide information about the context resources that can be linked to individual records; this is the case for Mali but not for Ghana. In our analyses of contraception, the effects of specific commodities differ widely and are not consistent upon different dimensions of the contraception culture and vary between men and women. For example, the presence of a radio station or a pharmacy in the neighbourhood does not influence the knowledge of contraception but, among women who declare to know modern contraception, it increases their use; such effect is not found among men.

- The place of residence as a proxy. In the countries studied here, the distinction between rural and urban settings remains a 
major index of life styles and context resources. The example of the contraception study highlights the importance of controlling for individual traits in order to distinguish a real effect of the context from an effect due to the composition of the local population. On the other hand, when the research strategy has targeted sectors with specific and documented characteristics, such as the Bamako youth study, the sector of residence provides useful indications about the influence of the degree of environment sanitation, the wealth of infrastructures, the cultural and socio-economic composition of the population and their lifestyles.

\section{Country comparison: differences in}

\section{opportunities resulting from context}

resources and collective conversion factors.

The two West African countries considered differ in their socio-economic dynamics and their trajectory in the demographic transition. As recalled in the second paper of this volume, this is expressed through a series of indicators including the Human Development Index (HDI) and the Multi-Dimensional Poverty Index (MPI) - that situate in many instances Mali and Ghana in the extreme of the spectrum of West Africa. Although designed in the Capability philosophy to better grasp differences in opportunities rather than in achievements, $\mathrm{HDI}$ and MPI provide little information about the roots of unequal development. A more promising way in the Capability literature is to consider the differences in terms of distinct "collective conversion factors ", such as applied in Hobson (ed, 2014) by the role of the work culture as regards differences in work-life balance among European countries. The differences between countries are therefore not only analysed through the national context resources, but also in the way these resources (institutional and cultural) interact with the collective capacity to convert them in wellbeing.

The case studies presented here were not designed for a systematic comparison between the two countries, but they highlight in several ways how the context resources and the ability to use them interact in both settings and give insights about similarities and dissimilarities. The notion of "collective conversion factor" is explicitly mentioned in von Jacobi's paper, whereas it is implicit through the notion of "contraceptive culture" in papers 9 and 10, and in differences in gender values as a transversal dimension. Von Jacobi analyses the intensity and type of social participation as a collective conversion factor that modulates women's employment security and its association with fertility achievements. While in both countries a context of higher social participation is associated with increased female secure employment, its effect on the combination of employment with large families differs. In Ghana, greater social participation appears to facilitate the family-work combination, while in Mali it is the opposite. This is interpreted as the consequence of less structural opportunities for Mali women: lower participation level and overall employment security (mainly in the agriculture sector), and high fertility. These structural dimensions of the context resources appear to be tightly linked to gender norms in social participation, as showed by a wider gender gap in participation in Mali, and an opposite effect of the relative participative level of women compared to men. In Mali, a higher share of female participation is associated with a higher baseline level of employment security, but it is enhancing the substitution effect between job and large family. The opposite is true in Ghana.

Differences in values are also illustrated by the comments of the interviewees in our qualitative surveys about roles and costs of children, the strength of the links with the family of origin and the negotiating within couples. All show well that Ghana is adopting urban behaviour associated with a trend towards individuation, while in Mali the role of the family and of traditions appear to remain more fundamental, especially among the women working in the agriculture sector.

However, our overall results about the factors influencing the adoption of a "contraceptive culture" and the ability to Shaping the family do hold for both countries. In both cases, inequalities in human resources explain the higher "contraceptive culture" of the large-city dwellers and longer birth 
intervals. It is not the fact of living in a large city, or of using contraception that counts, but the personal characteristics of the urban dwellers and of the contraception users. In this regards, the level of achieved education plays a clear role in both countries. Yet, the overall lower socioeconomic level of Mali is reflected in the fact that only the wealthiest households appear to have an advantage in terms of contraception use, in comparison with a gradient in Ghana.

The fact that, in Mali, family planning services appear to still discriminate against nonmarried individuals, whereas this is not the case in Ghana, may be the expression of the adherence to more traditional norms in this respect. Yet our results show that, not only in Mali, but in both countries, socio-cultural values and norms are playing an important role in the unequal ability to Shaping the Family. This is expressed by the net effect of ethnicity in both countries, but also by evidences as regards gender values and familialism. In spite of the differences in economic level, in both countries men's family plan is expressed through the ideal family size whereas women's are anchored into the reality of the actual number of children. In Ghana, such as in Mali, the adhesion to familialism is expressed by the anticipation of a large family through shorter birth intervals.

As regards health, the case studies display some similar results in both countries. Results highlight the adverse impact of motherhood on women health. They show that, even after controlling for characteristics of the population, the close surroundings per se have a significant influence on health outcomes. In both countries, the results also confirm that use of the services has not become a systematic behaviour. Among Accra women, it is rather frequent but more among wealthier households and apparently restricted to more serious health problems. Among Bamako youth it is much less frequent, not a characteristic of the better off, and for both sexes associated with contacts out of the household, i.e. having been to school and being professionally active; moreover; a first contact with the modern system diminishes the probability to use them at a subsequent health problem and does not erase inequalities of access. Therefore, in this type of population, under the present

circumstances, it does not seem to be legitimate to consider the use of services as a Capability, i.e. a dimension of the freedom to achieve good health.

In conclusion, more than the distinct levels of development, per se, the gap between these two countries has to be understood through the set of factors which model multi-sided processes towards modernization, but also the persistence of old values, and their implications for behaviour resistance and change. In a domain as intimate as family projects, the level of economic development such as traditionally measured has to be contextualized through unequal individual competence resulting from differentials in access to resources and unequal ability to use them and to negotiate the sociocultural norms.

\section{Tracking inequalities: Ongoing challenges in applying the CA to population studies}

The CA is providing clues to population studies to structure and refine the understanding of the sources of vulnerability. At this point, three promising issues are coming out of this research project that would be worth further developments. The two first refer to the challenge of distinguishing the different types of sources of inequalities, and the third one about the way to take in account individual decision making.

\section{Inequalities within and between groups: endowment groups and residual heterogeneity.}

Multivariate analysis provides tools to hierarchize the explanatory factors as regards the strength of their influence on a certain situation, and analysis by blocks have been used to highlight correlations between factors. This first step of the analyses provides clues to identify what we have called "endowment groups", that is to say people who share similar characteristics. In a second step, therefore, we proceeded to analyses disaggregated by such groups identified by their wealth, their level of schooling, gender, social participation or contraceptive culture. The intriguing question, one among the key issue of the CA, is however "Why [these] people with apparently the same 
opportunities end up in different situations?" Why is higher education, being wealthy or having a strong social network, not enough to erase inequalities? This leads to consider these endowments rather as means, combined with others. Education is not enough to erasing inequalities due to gender, or to a rural residence, in the capacity to use the skills learned and access to a secure job. Wealth is not enough to securing a good health, and contraception to having a smaller family, because other factors influence life styles and values, including everyday constraints. The analysis of these "remaining" sources of inequalities allows highlighting crosscutting issues such as gender, but also different residual sources of inequalities among women and among men. We have seen, for example, as for the Bamako young people, that cultural origin plays a role in male's health whereas environment sanitation is an important factor as for female's. One major challenge remains thus to better systematize this between/within groups analyses, in other words how to identify the main "endowment groups" and the remaining sources of inequality.

\section{Supply versus demand side: mitigated by social norms.}

The Capability framework brings in a distinction with major policy implications, that is between sources of inequalities due to the lack of adequate context resources - including services - and of personal resources that make people access and use them (conversion factors). The concept of Capabilities however, goes beyond a simple distinction between supply and demand as it implies that the use of the resource context should be oriented towards a life target which in turn is influenced by values, preference and constraints. The motivations to use the services have thus to be introduced as a third dimension. In a similar way, a triangular scheme is to be found in Coale's Ready-Able-Willing (RAW) model for behaviour change ${ }^{3}$. The supply side is distinguished by the ability to access resources including services - which beyond availability, includes their quality and costs. On the demand side, the focus is on the person's willingness to seek services, that is to say his/her degree of motivation with respect to anticipated benefits. Readiness introduces the socio-cultural background that makes the change in behaviour acceptable.

Both the CA and the RAW model adopt the actor's viewpoint by considering means and reasons for action and for behaviour modification. Both propose a triangulation between supply, demand and socio-cultural norms. The RAW model treats it by considering interactions between the availability of services and the person's perception of its quality, in the context of accepted norms and individual situations. Capabilities, seen as "substantive freedoms or opportunities that are created by a combination of the abilities residing inside a person (like capacities or skill) with their social, economic and political environment" (Nussbaum, 20II) provide a powerful tool to integrate these three dimensions. Both ways have proved to be very useful to build explanatory schemes of heterogeneity in behaviour change which are closer to the complexity of reality. Yet in both cases what remains unsolved is how to define precisely each parameter to operationalize the framework in demographic analysis and the construction of population policies.

\section{Agency and structure: ambivalence as a condition for innovative behaviour.}

Agency is a crosscutting dimension of the CA. "Freedom to achieve" can only be understood by considering the person as an active agent throughout the life course. Yet, the degree of freedom is unequally shared because agency is an embedded process in peoples' resources to cope with everyday contingencies as well as their position towards the prevailing social norms. Agency is shown up through the role played by the effect of differential in human resources on well-being. This has been demonstrated in our results as regards the influence of education, wealth or network. However, we have seen that inequalities are also to be found among those who enjoy the same level of resources. This is due in part to the combined effect of different types of characteristics. But most of all, as recognized by the $C A$, one must take in account 
personal preferences to understand "What enables people to choose the life one has reason to value?". In other words, "what matters most to the individuals concerned, rather than the relevance to researchers and policy makers" (Ariana and Naveed, 2009). This means to consider not only the etic view point the measurable aspects that are validated among the researchers - but also the emic side - what makes sense for the people themselves (Headland et al, eds, 1990). The etic approach may provide information on the emic side, for example through differentials in the role of personal resources. Yet it is not enough and models are still needed to better circumscribe what is meant in the CA by the still controversial notion of choice.

The notion of choice is misleading at least in two ways: it gives an illusion of rationality and of individual freedom unfettered by constraints. A series of attempts have been made to consider the role of agency and to model intentions and decision making ${ }^{4}$ in social science, including demography and public health. Yet what remains unsolved is how to consider the complex intermingling of personal agency with structures, that is to say the characteristics of the context, such as material obstacles or resources as institutions, rules or values. We have seen here that contextual resources have complex effects and that proxies for cultural settings such as ethnicity or religion can provide some insights about the kind of Capabilities that are influenced by values.

Yet, in this respect, it is necessary to consider the emic view point. As stressed by Hobson (20l4), this means to consider choice in reference to the array of actual opportunities, as perceived by the person herself, i.e. the "level of expectation bound to the perception of alternatives". In this regards, the framework offered by the concept of ambivalence appears promising. Applied by Lüscher and colleagues in the field of intergenerational relationships (see for example Lüscher, 20II), ambivalence expresses, in its simplest form, the simultaneity of dualistic notions, ideas, attitudes or emotions. This dualism can be shown by the gap between prevalent socio-cultural norms and a person's own life project. The concept of ambivalence provides thus a framework for measuring personal preferences against realities and ideal of normality (Lüscher, 20II; SauvainDugerdil 2005). Ambivalence is thus seen as a condition for innovative behaviour. Most traditional sectors of the population do not question the norms; they are not ambivalent and will not be actors for change. Yet, although a necessary condition, ambivalence is not sufficient in itself: new behaviours are carried only by individuals who have the personal resources that enable them to conceive and realize their personal plans.

The concept of ambivalence has been applied in the present project as regards obesity and family planning through qualitative surveys especially designed to analyse the gap, and therefore the ambivalence, between dominant values and personal preferences. This ambivalence is especially obvious as refer the conflict that many persons face between an overall valorisation of a large body size and personal views, especially of young progressive women, about aesthetic criteria and agility in everyday life. An important point raised by as regard the ability to shaping the family is that little ambivalence is to be found at the start of family life: the norm of having quickly the first child is not questioned. On the other hand, the gaps between contraception knowledge and use, and between the adoption of a contraceptive culture and the reality of birth spacing, is expressing conflicting positions between new and old norms. Further analyses are necessary to identify what makes some persons able to overpass the ambivalence to conceive and "live the life they have reason to value".

\section{Pointers for the future.}

This collection of papers represents the most ambitious attempt so far to take a very broad concept of the determinants and patterns of human well-being and to apply this to empirical socio-demographic data from West Africa. For sceptics, the choice of West Africa may seem curious but it makes sense on several levels. On the one hand, the availability of materials to assess the value of the Capability approach is about the same in West Africa as in other parts of the continent. On the other 
hand, there are for sure institutions and systems which are particular to the West African scene - the case of matrilineal descent amongst the Akan of Ghana being just one example - but in many other instances, the problems of weak state organisations and social security systems and poverty are common in many parts of the world.

For demographers, one of the salient conclusions from this body of work is the attraction of beginning with the consideration of processes rather than outcomes. The nearclassic approach based on the examination of an outcome, be it childhood mortality or fertility, and then working back through a series of proximate to ultimate determinants, is widely used but has been shown to deliver rather limited amount of information about the drivers of mortality and fertility change. Unlike the proximate determinants framework of Bongaarts (1982) and Mosley and Chen (2003), neither the outcomes of interest nor the proximate determinants are predetermined as several illustrations in the collection have shown. Depending on the level of analysis, functionings on one level may serve as Capabilities at another level of analysis.

In the main, current demographic analysis has focused very heavily on some of the technical problems of measuring cause and effect in non-experimental situations. This has led to considerable statistical sophistication and the borrowing of methods from cognate disciplines such as econometrics and spatial statistics. While all well and good at the level of doctoral training, for broader applications to policy and the setting of priorities for investments in social welfare broadly defined, the studies rarely provide a clear enough pathway between the intervention and desired outcome for our government department to use as a basis for its investment priorities. Frequently, the field of demography is regarded as atheoretical with a heavy dependence on clever analysis of empirical data. There have been a few attempts to provide a more theoretical framework, the most recent being the work of Johnson Hanks and colleagues (20II). Their work, a blend of sociology and social anthropology, hints at the possibility of the plasticity of concepts and measures elsewhere taken as fixed.

Until such time as a more thorough going and comprehensive general theory emerges about the nature of demographic behaviour and the determinants of human well-being, it seems to us that the Capability approach does open a much overdue theoretical debate not only about causes and consequences but also about values and norms. Although well-being has often been reduced to a few simple quantitative measures such as those produced by the UN Development Programme in the form of the Human Development Index and the many indicators developed as part of the Millennium Development Goals, there is now room for a broader debate about the meaning of wellbeing in different wealth contexts. The nonlinear association between per capita incomes, or other measures of wealth, and most objective measures of satisfaction or confidence or happiness conducted by major polling organisations, suggests that other interpretations of well-being are necessary to capturing important differences between and within human societies.

We hope that this collection of papers stimulates some members of the upcoming cohorts of demographers and social scientists in Africa to think more broadly about the determinants of human well-being and success in their own societies. We hope to that the difficulties laid bare by the authors in producing coherent accounts of the reasons for the inequalities in different societies using existing data which generally are short on preferences and values encourage new generations of analysts to think both originally and creatively about the sources and drivers of inequalities in their own societies.

\section{References}

Ariana, Proochista and Arif Naveed (2009), Health. Chap 10 in Deneulin and Shahani (eds): An Introduction to the Human Development and Capability Approach. Freedom and Agency. London, Earthscan.

Basu, K (20I3), Group identity, productivity and well-being: policy implications for promoting 
development. J Human Devel and Capabilities 14(3), 323-40.

Billari, Francesco, Dimitar Philipov and Maria R.

Testa (2005), The influence of attitudes, subjective norms and perceived behavioural control on union formation intentions. IUSSP Conference Tours.

Bongaarts, John (1982), The fertility-inhibiting effects of the intermediate fertility variables. Stud Fam Plann I3(6-7), I79-I89.

Bonvalet, Catherine et Eva Lelièvre (dir. 20I2), De la famille à l'entourage. L'enquête biographies et entourage, INED, coll. "Grandes Enquêtes ".

Coale, Ansley (1973), The demographic transition reconsidered, In IUSSP, Proceedings of the International Population Conference. Liège, Eds. Ordina, 53-73.

Deneulin, Séverine and Lila Shahani (eds, 2009), An Introduction to the Human Development and

Capability Approach. Freedom and Agency. London, Earthscan.

Emirbayer, Mustafa and Ann Mische (1998), What is Agency? American J of Sociology 103(4), 962-1023.

Guilmoto, Christophe Z (2009), The sex ratio transition in Asia. CEPED, Paris. Working Papers 2.

Headland Th. N., K.L. Pike and M. Harris (eds, 1990), Emics and Etics. The Insider / Outsider Debate. Frontier of Anthropology, V. 7. Summer Inst. of Linguistics, Sage.

Hobson, Barbara (ed, 20I4), Worklife Balance. The Agency and Capabilities Gap, Oxford University Press.

Johnson-Hanks, J. (2006). Uncertain honor: modern motherhood in an African crisis. Chicago, The University of Chicago Press.

Johnson-Hanks, Jennifer, Christine Bachrach, Philip Morgan and Hans-Peter Kohler (20II), Understanding Family Change and Variation: Toward a theory of Conjunctural Action. Springer.

Lesthaeghe, Ron and Camille Vanderhoeft (200I), Ready, Willing, and Able: A Conceptualization of Transition to New Behavioral Forms. In Diffusion Processes and Fertility Transition: Selected
Perspectives, John B. Casterline (ed), The National Academies Press, 240-264.

Lesthaeghe, Ron (1995), The second demographic transition in western countries: an interpretation. In Karen Oppenheim Mason and An-Margitt Jensen : Gender and Family Change in Industrialized Countries, Oxford University Press, 17-62.

Lindenberg, Siegwart (1996), Continuities in the theory of social production function. Reprint from H.Ganzeboom and S.Lindenberg (eds): Verklarende Sociologie: Opstellen voor Reinhard Whiple. Thela, Amsterdam.

Lüscher, Kurt (20II), Advancing the field ambivalence : A "sensitizing construct" for the study and practice of intergenerational relationships. J. of Intergenerational Relationships, $9: 191-206$.

Mosley, W. H. and L. C. Chen (2003), An analytical framework for the study of child survival in developing countries. 1984. Bull World Health Organ 8I(2), I40- 145.

${ }^{1}$ In the same way the use of contraception is considered for the former as a resource and the latter a means.

${ }^{2}$ Average schooling, structure of the labor market, rate of women not active professionally, percent women head of household, indicator of women empowerment, community activism, ethnolinguistic heterogeneity, wealth index (average and Gini index of dispersion), available infrastructures.

${ }^{3}$ This model, developed to explain fertility decline in Europe, has been reformulated by Lesthaegue and Vanderhoeft (2001), and recently applied by Guilmoto (2009) in reference to sex selection in Asia.

${ }^{4}$ In demography, in particular, work done by colleagues who adapted Ajzen Theory of Planned Behaviour to family analysis in the Gender and Generation Program (Billari et al 2005; Rossier and Bernardi, 2009), 